\title{
Absorption and elimination of formate following oral administration of calcium formate in female human subjects
}

Publisher version available: http://dmd.aspetjournals.org/content/33/2/282.full.pdf

Author's accepted manuscript, available through PubMed Central:

http://dmd.aspetjournals.org/content/early/2004/11/16/dmd.104.001289

\section{Full Citation of published version:}

Hanzlik RP, Fowler SC, Eells JT. Absorption and elimination of formate following oral administration of calcium formate in female human subjects. Drug Metab Dispos. 2005 Feb; 33(2):282-6.

\section{ABSTRACT:}

Calcium formate is a water-soluble salt of an essential mineral nutrient with potential for use as a dietary calcium supplement. Formate ion is a product of endogenous and xenobiotic metabolism, but sustained high plasma formate concentrations (such as occur in cases of methanol poisoning) are toxic to the retina and optic nerve. Humans and primates have reduced capacity for formate oxidation compared with rodents and dogs and are thus more sensitive to methanol (and formate) intoxication. To assess the potential for accumulation of formate ion upon repeated administration of calcium formate as a potential dietary calcium supplement, we measured plasma concentrations of formate in 14 adult human subjects before and after oral administration of a single large dose of calcium formate (3900 mg; ca. 3-6 times the anticipated dose for calcium supplementation). Plasma formate concentrations increased briskly from $0.024 \pm 0.008 \mathrm{mM}$ (endogenous formate) to reach $\mathrm{Cmax}(0.50 \pm 0.04 \mathrm{mM}$ ) at 60 min postdose and then declined with a half-life of $59 \pm 7 \mathrm{~min}$. By $225 \mathrm{~min}$ postdose, plasma formate concentration had returned to baseline. With such a short half-life, repeated use of calcium formate as a dietary supplement, even three times daily, should not lead to progressive accumulation of formate. These findings are discussed in light of the production of formate by endogenous and xenobiotic metabolism and the kinetics of formate during methanol poisoning.

Departments of Medicinal Chemistry (R.P.H.) and Pharmacology and Toxicology (S.C.F.), University of Kansas, Lawrence,Kansas; and the Department of Health Sciences, University of Wisconsin-Milwaukee, Milwaukee, Wisconsin (J.T.E.)

Accepted November 15, 2004 